\title{
Defect Study in Fused Silica using Near Field Scanning Optical Microscopy
}

\author{
M. Yan \\ L. Wang \\ W. Siekhaus \\ M. Kozlowski \\ J. Yang \\ U. Mohideen
}

This paper was prepared for and presented at the 29th Annual Symposium on Optical

Materials for High Power Lasers

Boulder, Colorado

October 6-8, 1997

January 21, 1998

This is a preprint of a paper intended for publication in a journal or proceedings. Since changes may be made before publication, this preprint is made available with the understanding that it will not be cited or reproduced without the permission of the author. 


\section{DISCLAIMER}

This document was prepared as an account of work sponsored by an agency of the United States Government. Neither the United States Government nor the University of California nor any of their employees, makes any warranty, express or implied, or assumes any legal liability or responsibility for the accuracy, completeness, or usefulness of any information, apparatus, product, or process

disclosed, or represents that its use would not infringe privately owned rights. Reference herein to any specific commercial product, process, or service by trade name, trademark, manufacturer, or otherwise, does not necessarily constitute or imply its endorsement, recommendation, or favoring by the United States Government or the University of California. The views and opinions of authors expressed herein do not necessarily state or reflect those of the United States Government or the University of California, and shall not be used for advertising or product endorsement purposes. 


\title{
Defect Study in Fused Silica using Near Field Scanning Optical Microscopy
}

\author{
Ming Yan, Li Wang, Wigbert Siekhaus and Mark Kozlowski \\ Lawrence Livermore National Laboratory \\ P.O. Box 808 , L-250 \\ Livermore, CA 94551 \\ Jason Yang, Umar Mohideen \\ Department of physics, University of California \\ Riverside, CA 92521
}

\begin{abstract}
Surface defects in fused silica have been characterized using Near Field Scanning Optical Microscopy (NSOM). Using total internal reflection of a p- or s- polarized laser beam, optical scattering from defects located on the surface itself as well as in the subsurface layer of polished fused silica has been measured by NSOM. The local scattering intensity has been compared with simultaneously measured surface topography. In addition, surface defects intentionally created on a fused silica surface by nano-indentation have been used to establish a correlation between optical scattering of s- and p-polarized light, surface morphology and the well known subsurface stress-field associated with nano-indentation.
\end{abstract}

Keywords: near field scanning optical microscopy, defects, nano-indentation, fused silica.

\section{Introduction}

With the increasing emphasis on nano-fabrication technologies, the nano-scale physical properties of materials have received intense interest. Even at macroscopic length scales, the properties of materials are often dominated by local, sub-micron-scale structures which, in many cases, are formed by defects. The importance of local properties is dramatically revealed by the process of laser induced damage in optical materials where damage is typically initiated at the submicron scale. In recent years, atomic force microscopy (AFM) has been used to investigate surfaces of materials at sub-micron length scales and to identify surface morphologies that initiate laser damage $^{1}$. However, AFM provides no information about local optical properties or chemical speciation. The emergence of near field scanning optical microscopy (NSOM) and spectroscopy (NSOS) makes it now possible to observe both physical structure and optical properties ${ }^{2-9}$.

\section{Experimental setup}

The scanning head of the NSOM instrument, shown in the left image of figure 1, is based on commercially available scanning electronics of an atomic force microscope. The NSOM instrument uses a pulled fiber tip with an aperture of about $100 \mathrm{~nm}$, as shown in the center and right images of figure 1, for illumination of the sample or collection of optical signals, such as transmitted or fluorescent light. The spatial resolution of the instrument is determined by the diameter of the fiber tip, which is much smaller than the optical wavelength. With it we have achieved topographic resolution close to that of an AFM and have used it to study the correlation between surface morphology and associated subsurface strain and s- and p- polarized light scattering from polishinginduced and from intentionally produced surface defects on fused silica surfaces. 

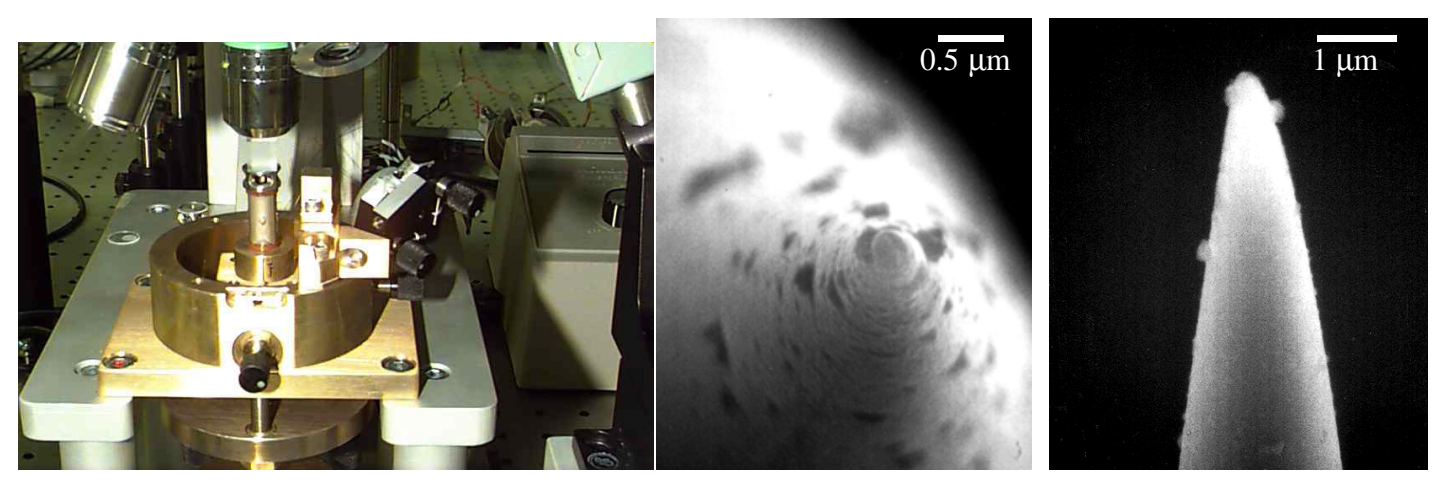

Figure 1. The scanner of NSOM instrument (left) and the top view (middle) and side view (right) a fiber tip for light collection.

\section{Characterization of polishing defects in fused silica}

The process of polishing fused silica results in a contaminated surface layer $\sim 50-100 \mathrm{~nm}$ thick. This surface layer has been studied in the past by total internal reflected light scattering microscopy ${ }^{10}$ and atomic force microscopy. However, the first technique provides only low spatial resolution $(>10 \mu \mathrm{m})$ optical and surface topographic information, while the second technique only provides surface topography at the nanometer scale. To combine the optical scattering measurement with scanning probe microscopy, we used NSOM to collect scattered light from a totally internal reflected incident beam while monitoring simultaneously surface topographic defects. The near field optics collects the scattered light only from the top $100 \mathrm{~nm}$ beneath the surface. Thus defects in the contaminated layer are imaged. Figure 2 shows side by side a topographic image and a total internal reflection (TIR) image. The TIR image maps light scattered from defects under conditions where a perfect surface would have reflected all light away from the detector. Comparison between the two types of images makes it clear that the optical scattering NSOM image (left) reveals information one cannot see in the NSOM surface morphology image (right). This strongly suggests that we can image subsurface defects.
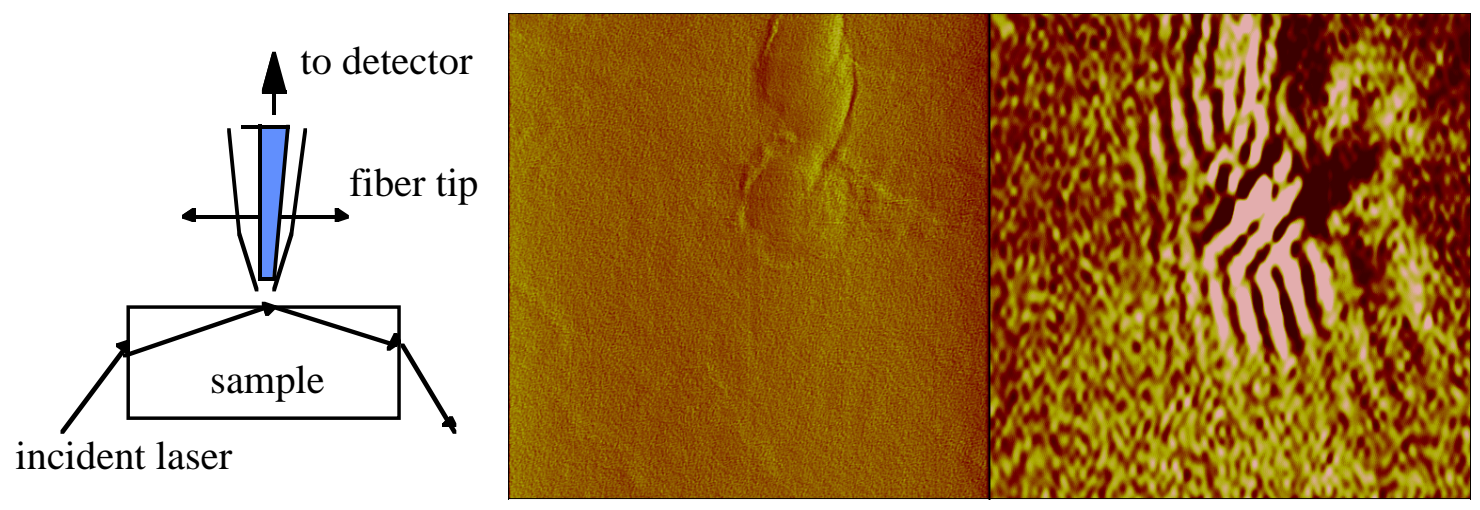

Figure 2. Sketch of total internal reflection NSOM, and topographical (left) and optical scattering

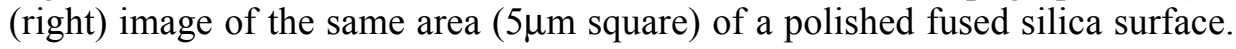


Since the fiber tip is held less than $10 \mathrm{~nm}$ above the fused silica surface, the evanescent wave from the surface is also collected by the fiber tip due to the optical coupling of the surface to the optical fiber. Such evanescent waves are sensitive to surface and sub-surface defects in the collection depth of the near field optics. By changing the polarization of the total internal incident light, the sub-surface and surface features can be distinguished due to the difference in the distribution of nearsurface and on-surface optical fields for the two polarizations.

\section{Characterization of nanoindentation defects in fused silica}

To better understand the NSOM optical image, we have made intentional nanometer deep indentation--defects on a polished fused silica surface using AFM-based nano-indentation with a diamond tip. Figure 3 shows a topographic AFM image which reveals that the artificial pits are $\sim 50 \mathrm{~nm}$ in width and $\sim 3 \mathrm{~nm}$ in depth. The topographic image obtained by the NSOM's fiber tip also measures the surface pits. However, the resolution is limited by the size of optical fiber tip. NSOM optical images clearly resolve the pits on the surface. Theory predicts that the maximum of the electrical field of the totally internally reflected beam with s polarization is on the surface while $p$ polarization is below the surface ${ }^{10}$. The optical image with two different polarizations agrees with the predicted electrical field distribution. Thus, for the first time, our experiment verifies this theory at the sub-wavelength scale. In addition, as figure 3 shows, the observed spatial size of optical scattering is much larger than the topographic size. This shows that the laser scattering is not limited to the indented area, but is also generated by the strained area produced when indenting the material. More careful analysis is ongoing to measure the stress associated with processing on the nano-meter scale.
AFM
NSOM
NSOM
NSOM
topographic image. topographic image. Optical image
Optical image
(p-polarization)
(s-polarization)

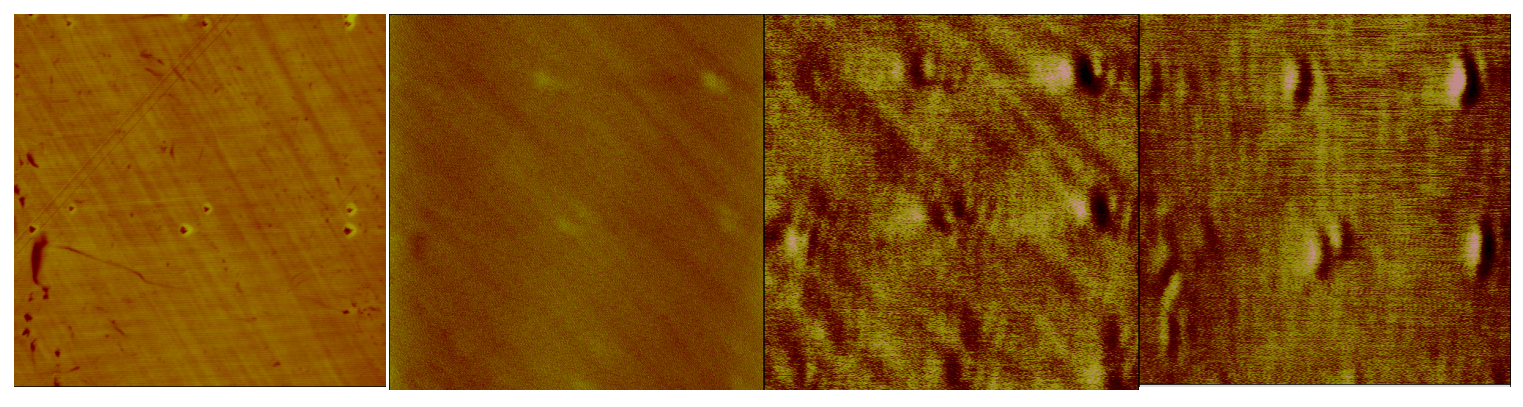

$1 \overline{\mu m}$

(a) (b) (c) (d)

Figure 3. AFM topographic(a), NSOM topographic(b) and NSOM optical images with s (c) and p (d) polarization in a nano-indented fused silica sample.

\section{Summary}

In summary, the local scattering intensity has been compared with simultaneously measured surface topography. Surface defects intentionally created on a fused silica surface by nanoindentation have been used to establish a correlation between optical scattering of s- and p- polarized 
light, surface morphology and the well known subsurface stress-field associated with nanoindentation. We have provided new optical information concerning accidental and intentional submicron defects on surfaces and sub-surfaces which is important for both ICF and industrial applications using NSOM.

\section{Acknowledgements}

We would like to thank Jim De Yoreo and Stan Oberhelman for helps and supports in this work. This work was performed under the auspices of the US department of Energy by Lawrence Livermore National Laboratory under contract No. W-7405-Eng-48.

\section{References}

(1) R.J. Tench, R. Chow, and M.R. Kozlowski, "Characterization of Defect Geometries in Multilayer Optical Coatings," Journal of Vacuum Science and Technology A 12/5, 2808 (1994).

(2) E. Betzig, J.K. Trautman, T.D. Harris, J.S. Weiner and R.L. Kostlak, "Breaking the Diffraction Barrier: Optical Microscope on a Nanometric Scale" Science, 251, 1468 (1991).

(3) J.J. Macklin, J.K. Trautman, T.D. Harris, L.E. Brus, "Imaging and time resolved spectroscopy of single molecules at an interface", Science, 272, 255 (1996).

(4)J.W. P. Hsu, E. A. Fitzgerald, Y. H. Xie, P. J. Silverman, "Near-Field Scanning Optical Microscopy Imaging of Individual Threading Dislocations on Relaxed GeXSi1-x Films", Appl. Phys. Lett. 65, 344 (1994).

(5) T.J. Yang, U. Mohideen and M.C, Gupta, "Near-field scanning optical microscopy of ferroelectric domain walls" Appl. Phys. Lett., 67, 1960 (1997)

(6) C. L. Jahncke, M. A. Paesler, H. D. Hallen, "Raman Imaging with Near-Field Scanning Optical Microscopy", Appl. Phys. Lett. 67, 2483 (1995).

(7) R.D. Grober, T.D. Harris, J. K. Trautman, E Betzig, "Optical Spectroscopy of a GaAs/AlGaAs

Quantum Wire Structure using Near-Field Scanning Optical Microscopy”, Appl. Phys. Lett. 64, 1421 (1994).

(8) H.D. Hallen, A. H. Larosa, C. L. Jahncke, "Near Field Scanning Optical Microscopy and Spectroscopy for Semiconductor Characterization", Phys. Stast. Sol. A, 152, 257 (1995).

(9) A. Jalocha, N.F. Vanhulst, "Polarization Contrast in Fluorescence Scanning Near-Field Optical Reflection Microscopy”, J. Opt. Soc. Amer. B 12, 1577 (1995).

(10) C.F. Kranenberg, K.C. Jungling, "Subsurface damage identification in optically transparent materials using a non-destructive method", Applied Optics, 33, 4248 (1994) 


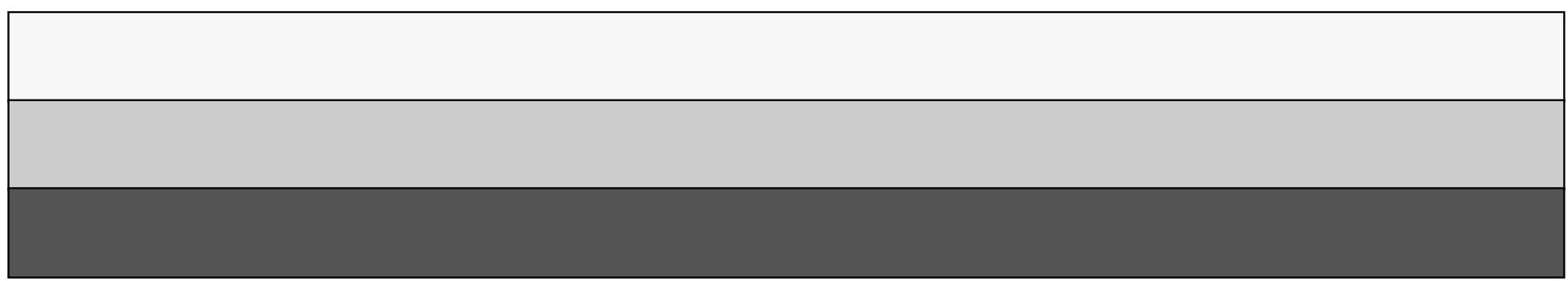

